\title{
Excess electron states in fluid methane: Density-functional versus Lanczos approaches
}

\author{
Fernan Saiz, Nick Quirke, Leonardo Bernasconi \\ and David Cubero
}

\section{Published version information}

Citation: Saiz, F et al. "Excess electron states in fluid methane: Density-functional versus Lanczos approaches." Chemical Physics Letters, vol. 664 (2016): 143-148.

DOI: $\underline{10.1016 / \text { i.cplett.2016.10.033 }}$

(C)2016. This manuscript version is made available under the CC-BY-NC-ND 4.0 Licence.

This version is made available in accordance with publisher policies. Please cite only the published version using the reference above. This is the citation assigned by the publisher at the time of issuing the AAM. Please check the publisher's website for any updates. 
Excess electron states in fluid methane:

Density-functional versus Lanczos approaches.

\author{
Fernan Saiz, Nick Quirke ${ }^{1}$ \\ Department of Chemistry \\ Imperial College, London
}

SW7 2AZ, UK

Leonardo Bernasconi

STFC Rutherford Appleton Laboratory, Didcot, Oxon, OX11 0QX, UK

\author{
David Cubero \\ Departmento de Física Aplicada I, \\ Universidad de Sevilla, \\ Calle Virgen de Africa 7, \\ 41011 Seville, Spain
}

\begin{abstract}
We compare density-functional theory (DFT) electronic structure calculations at the hybrid B3LYP level for fluid methane with experiments and a pseudo-potential Lanczos method. We generate fluid configurations from classical/ab initio molecular dynamics and use DFT to determine one-particle orbital and total energies. Our results show that DFT predicts excess electron energies that qualitatively agree with experiments over a wide density range, provided these values are determined from total energy differences between charged and neutral systems. By contrast, and contrary to previous claims, orbital energies of the lowest unoccupied state of the $\mathrm{N}$-electron system provide qualitatively incorrect excess electron energies as a function of the fluid density.
\end{abstract}

\footnotetext{
${ }^{1}$ Corresponding author: n.quirke.ic.ac.uk
} 


\section{Introduction}

The study of excess-electron states is of theoretical and practical interest. In dense fluids of small spherical molecules, the excess-electron mobility varies as a function of atomic density and exhibits a maximum at liquid densities ${ }^{1}$. This behaviour has been linked to the nature of the excess-electron wave-function: in fluids of symmetrical molecules, such as methane, the electron is delocalised at all fluid densities, whereas in fluids of asymmetrical molecules, such as ethane, the ground state becomes localised at liquid densities ${ }^{2}$, leading to lower mobilities. For alkanes and other fluids, theoretical predictions of the ground state energy of the excess-electron can be compared to the experimentally determined excess-electron lowest-energy conducting state $V_{0}$, which is taken as the bottom of the conduction band of the fluid $^{3,4}$.

In the solid state, the behaviour of excess electrons is key to understanding charged defects ${ }^{5}$, charge trapping ${ }^{6,7}$, polaron formation ${ }^{8,9}$ and charge transport ${ }^{10}$. The localisation of electrons in disordered materials was described 50 years ago by Anderson ${ }^{11}$, who linked the electron localisation length to the degree of disorder in the potential energy surface. The case of polyethylene is of particular interest, as it is a commonly used insulator for high-tension cables. The electronic properties of excess electrons injected into polyethylene - so-called space charge - are thought to be related to commercially important properties, such as dielectric breakdown and (high-tension) cable lifetimes ${ }^{12}$. For polyethylene, the properties of excess electrons have been studied using density-functional theory ${ }^{6,13,15}$ (DFT) and singleelectron approaches such as the Lanczos method ${ }^{4,14}$. DFT minimises the total energy with respect to variations in the electronic density to solve the $N$-electron Schrödinger equation, whereas the Lanczos method solves the Schrödinger equation for one electron in a pseudopotential, which for polyethylene was constructed by fitting it to the conduction band data $V_{0}$ of short chain alkanes., ${ }^{414}$ The Lanczos method represents the energy surface for an excess electron in the presence of $N$ electrons and their nuclei with all multi-electron interactions subsumed into the one-electron pseudo-potential. By contrast, DFT represents the individual electrons through a set of auxiliary independent-particle Kohn-Sham (KS) orbitals, which represent individual electrons in the presence of the mean-field potential generated by the other electrons. The Lanczos approach has been shown to provide reliable estimates of excess electron energies for fluid methane (and other molecules) over a wide range of densities ${ }^{2,3}$ In this work, we study the ability of the more computationally expensive, but potentially parameter free, DFT methods to address the calculation of the same quantities.

The excess electron is interpreted differently in the Lanczos method and in DFT. In previous work using DFT the excess electron energy has been calculated either from the energy of (KS) LUMO (bottom of the conduction band) of the neutral $N$-electron system ${ }^{6}$ or from the HOMO of the $N+1$ system $^{15}$. These two approaches are fundamentally different: in the former case the LUMO energy is assumed to approximate the energy of an electron added to the neutral $\mathrm{N}$-electron system, whereas in the latter an electron is physically added to the system. In the Lanczos method the excess electron is represented by the single-electron states of the direct solution of the Schrödinger equation. The problem of estimating excess electron energies in DFT is complicated by the strong dependence of these quantities on the approximation used to represent the exact exchange-correlation functional ${ }^{16}$. In this work, we will use the hybrid B3LYP approximation, which has been shown to give excellent estimates of ground and excited state properties (including band gaps ${ }^{17}$ and optical gaps ${ }^{18}$ ) of extended 
systems. The main advantage of DFT over pseudo-potential methods is its ability to fully represent the $N+1$ - and $N$-electron systems from first principles. DFT can be used in situations where a reliable pseudo-potential is difficult to build due to insufficient experimental or $a b$ initio data.

The interpretation of the LUMO energy of the $N$-electron system as an excess electron energy is questionable. In contrast to Hartree-Fock theory, the LUMO energy in DFT is not correlated to the electron affinity, and therefore the LUMO does not describe the distribution of an electron added to the system. The HOMO of the $N+1$-electron system can be rigorously identified in exact DFT with the electron affinity of the $N$-electron system ( $c f$. Figure 1 in Ref. Error! Bookmark not defined. and discussion therein), which provides an approximation to the true excess electron energy. The direct calculation of the HOMO energy of the $N+1$-electron system is straightforward for finite systems, whereas it gives rise to a number of technical complications in genuinely periodic systems ${ }^{41}$. In this work, we show that the KS orbitals are not able to predict correct excess electron energies however a difference of total energies of the ground state of the $N+1$ - and $N$-electron systems for the same atomic configuration does provide a qualitatively correct description of the dependence of the excess electron energy on the sample density. We will also propose a simple empirical correction for estimating accurate excess electron energies from DFT for wide ranges of densities. We use all-electron DFT calculations at the B3LYP level to estimate excess electron energies of fluid methane both from KS orbital energies and from the $N+1 / N$ total energy difference and we compare these results to Lanczos calculations employing an $a b$ initio pseudo-potential and to experimental values. We will also examine the nature and localisation properties of the excess electron at various densities.

\section{Methods}

We have employed the molecular dynamics package LAMMPS $^{19}$ to generate classical configurations of fluid methane. The methane systems are composed of 32, 64, 125, 216, and 343 molecules, with varying values of the reduced density $\rho^{*}=\rho \sigma^{3}$, where $\rho$ is the number density and $\sigma=3.923 \AA$ is the characteristic length associated with the Lennard-Jones interaction between methane molecules ${ }^{2}$. For the 64-molecule simulations, we consider systems with reduced densities between 0.1 and 1.0 in intervals of 0.1 . For the simulations with a different number of molecules, we only consider the densities $\rho^{*}=0.5$ and 0.9 . The interatomic forces are calculated with the explicit-hydrogen TRAPPE-EH ${ }^{20}$ potential, which provides an accurate description of liquid methane and its phase equilibria. The equations of motion are integrated for $2 \mathrm{~ns}$ at constant volume and a fixed temperature of $340 \mathrm{~K}$, and then for another $2 \mathrm{~ns}$ at constant volume and energy with a time-step of $1 \mathrm{fs}$. We then run short Born-Oppenheimer molecular dynamics simulations using the $\mathrm{CP} 2 \mathrm{k}^{21}$ package in its QUICKSTEP ${ }^{22}$ implementation. The purpose of this short simulation is to relax the C-H bond lengths, which have been fixed during the classical step. These calculations use the BLYP ${ }^{23,24}$ exchange-correlation functional, GTH pseudopotentials ${ }^{25,26}$, and double- $\zeta$ basis sets with polarization functions (DZVP). ${ }^{27} \mathrm{We}$ used a threshold of $3 \times 10^{-6} \mathrm{Ha}$, a cut-off of $350 \mathrm{Ry}$ for the plane wave basis set, and the scheme DFT-D2 ${ }^{28}$ to include long-range forces. 
The configurations resulting from the Born-Oppenheimer molecular dynamics step are then used by the Lanczos algorithm and the CRYSTAL14 DFT code ${ }^{29}$. The DFT calculations are carried out at the $\mathrm{B} 3 \mathrm{LYP}^{30}$ level using periodic boundary conditions. A standard all-electron $6-31 \mathrm{G}^{* * 31,32}$ basis set is used to represent the local atomic orbitals in terms of primitive Cartesian Gaussian functions. Polarization functions ( $\mathrm{p}$-functions for hydrogens and $\mathrm{d}$ functions for carbons) are included to ensure that the orbitals can distort from their original atomic symmetry, and to better adapt to the molecular surroundings leading to a better prediction of the total energy of the system with high hydrogen content. Dispersion interactions are accounted for using the DFT-D2 method ${ }^{33}$. Reciprocal space integrations are restricted to the $\Gamma$-point of the Brillouin zone and a ground-state energy convergence is enforced at $1 \times 10^{-6} \mathrm{Ha}$. Standard parameters for two-electron integral calculations are used throughout. The calculations are carried out for the $N$ - and the $N+1$-electron systems using the same atomic configuration and simulation cell parameters. In the $N+1$-electron systems, a positive homogeneous charge background is added to avoid divergences in the total energy originating from the periodic boundary conditions.

\section{Results}

Figure 1 shows the KS orbital energies as a function of reduced molecular density for methane containing $N$ and $N+1$ electrons and compares them to the lowest-energy conducting state $V_{0}$ of the excess-electron determined experimentally. We identify this state as the bottom of the conduction band of fluid methane. The LUMO of the $N$-electron system, which represents the bottom of the KS conduction band, exhibits a marked qualitative difference with the experimental behaviour. The same is true for the HOMO of the $N+1$ system. The values at the lower densities are far too large, and the rate of change with density is qualitatively incorrect. Unlike the experimental results, the KS energies show no minimum in the conduction band. Intriguingly, the $N+1$-electron system HOMO does agree with the experiment in a small region of liquid densities around $\rho^{*}=0.9$. For this reason, we believe that excess electron energies obtained from KS orbital energies may be used, as proposed in the literature, but only over a very restricted density range. We also show in Figure 1 the ground state of the Lanczos excess-electron system, which is in excellent agreement with experiment at all densities. Strikingly, these latter theoretical predictions are obtained with a pseudo-potential derived fully from $a b$ initio calculations ${ }^{2}$ namely $l=0$ phase shift scattering for the repulsive and short-ranged component of the excess-electron-molecule interaction, and polarisability calculations for the attractive and long-ranged part. Furthermore, the Lanczos virtual states are very close in energy, differing by only fractions of an $\mathrm{eV}$, at variance with the DFT orbital energies. The data in Figure 1 corresponds to the $6-31 \mathrm{G}^{* *}$ basis. Since we expect the electron to be fully delocalised in methane, we have also tested a more diffuse basis set, $6-31+\mathrm{G}$, which is found to shift the HOMO of the N+1-electron system to negative values $\left(\approx-2 . \mathrm{eV}\right.$ for $\left.\rho^{*}=0.8\right)$, but it does not improve the dependence of the energy on the density. In particular, no minimum is observed in the orbitals energies.

We now study the variation of the orbital energies with the excess electron density to minimise any image effects of the excess-electron due to the use of periodic boundary conditions. By increasing the number of methane molecules at constant density, the excesselectron concentration is varied between 0.26 and $0.024 \mathrm{~nm}^{-3}$. Figure 2 shows that the HOMO becomes slightly more positive at $\rho^{*}=0.5$, whilst the LUMO drops by $0.4 \mathrm{eV}$, making the band gap HOMO-LUMO much smaller. However, the position of the HOMO remains in 
disagreement with experiment. It is interesting that all the gaps between states become significantly smaller as the excess-electron density decreases, qualitatively approaching the behaviour predicted by the single-electron model of the Lanczos method. We observe a similar behaviour at higher methane densities. Clearly, the KS orbital energies in the $N$ or $N+1$-electron systems are not representative of the experimental properties of excess electrons in fluid methane for all densities.

Bearing in mind that DFT minimises the total energy with respect to the $N$ electron density, we now investigate whether a better description of the excess electron energy can be obtained from the difference between the total energy of the $N+1$ and $N$-electron systems. This difference is labelled as $\varepsilon_{\mathrm{gs}}$ to represent that is calculated from ground-state calculations on the $N+1$ and $N$-electron systems with no input from KS orbital energies,

$$
\varepsilon_{\mathrm{gs}}=E_{\mathrm{TOT}}(N+1)-E_{\mathrm{TOT}}(N) .
$$

Although the absolute values of the excess energy disagree with experiments, Fig. 3 shows that the variation of $\varepsilon_{\mathrm{gs}}$ with the density reproduces the experimental behaviour for reduced densities $\rho^{*}$ larger than 0.3 , where $\rho^{*}=\rho \sigma^{3}$, being $\rho$ the nuclear number density and $\sigma$ the Lennard-Jones parameter used in the classical methane simulations. At low densities, we believe that the mismatch in the trend of DFT and experimental energies can be attributed to the inability of the $6-31 \mathrm{G}^{* *}$ basis set to provide reliable estimates of the electron affinity near the molecular $\left(\rho^{*}=0\right)$ limit. ${ }^{34}$ Nevertheless, the quality of this basis set is satisfactory in the intermediate and high-density regimes, possibly due to the contraction of the orbitals of individual methane molecules in the condensed-phase environment.

Figure 4 shows the electron affinity obtained by CRYSTAL14 for one methane molecule $E A$ $=E_{\mathrm{TOT}}(N+1)-E_{\mathrm{TOT}}(N)$ as a function of the reduced density and the basis sets 6-31G** and 6$31+\mathrm{G}$, which includes diffusive functions to generate significant electron density at relatively large distances from the nucleus during the self-consistent iterations. The methane molecule is placed in a simulation cell with periodic boundary conditions, whose volume is adjusted such that the reduced atomic density $\rho^{*}$ varies from 0.1 to 1.0 . The data point at $\rho^{*}=0.0$ corresponds to a calculation carried out without periodic boundary conditions, i.e. on an isolated molecule in vacuum. As the box length $L$ increases, the EA converges rapidly to a value of $-5.6 \mathrm{eV}$, whereas the value expected from higher level calculations [MP2 and $\operatorname{CCSD}(\mathrm{T})$ from Ref. 34] is zero. This confirms that in our calculations the 6-31G** basis set fails at very low densities in the $N+1$ system, as the diffuse orbitals of the methane anion are incorrectly accounted for. In order to compute reliably $\varepsilon_{\mathrm{gs}}$ at very low densities, more diffuse basis (or floating/plane waves) sets will have to be used. The computational cost associated with the large number of plane waves required to model the electron densities of systems approaching the molecular regime is however much larger that with a local Gaussian basis set. Furthermore, calculations with plane waves may become prohibitively expensive with hybrid DFT functionals ${ }^{35,36,37}$. Nonetheless, Table I collects the electron affinities at $\rho^{*}=0.0$ calculated with more diffuse basis sets than $6-31 \mathrm{G}^{* *}$. Our results show that the disagreement obtained with $6-31 \mathrm{G}^{* *}$ with respect to the experiment is dramatically reduced when a diffusive function is included by using 6-31+G. In addition, the polarization behaviour of $3 \mathrm{~d}$ functions in $6-31+\mathrm{G}^{* *}$ have little impact on the electron affinity since this value increases by only $0.2 \mathrm{eV}$. Furthermore, the agreement with experiment is still improved by $1.01 \mathrm{eV}$ when we add a second diffusive function with $6-31++\mathrm{G}^{* *}$. The addition of more complex basis sets 
6-311++ $\mathrm{G}^{* *}$ and $6-311++\mathrm{G}^{* *}(2 \mathrm{~d}, 2 \mathrm{p})$, which adds two $\mathrm{d}$ and $\mathrm{p}$ more diffusive functions than the former, has a negligible effect on the electron affinity. Therefore, we conclude that our DFT simulations with B3LYP predict an electron affinity of $-0.92 \mathrm{eV}$ with an extrapolated infinite basis set.

As observed, the calculated $\varepsilon_{\mathrm{gs}}$ dependence on the fluid density mirrors the experimental excess electron energy behaviour, although the absolute value of $\varepsilon_{\mathrm{gs}}$ turns out to be higher than the experimental (and Lanczos) estimates. In practice, we find that a quantitative measure of the excess electron energy is given by $\varepsilon_{\mathrm{gs}}+c$, where $c=-2.63 \mathrm{eV}$, with a root mean square deviation (RMSD) of $0.03 \mathrm{eV}$ from the experimental data for $\rho^{*} \geq 0.3$ (in contrast to our Lanczos predictions which have an RMSD of $0.014 \mathrm{eV}$ for $\rho^{*} \geq 0.2$ ). We attribute this difference between DFT and experimental excess electron energies to the residual self-interaction error (SIE) of the B3LYP functional, which affects the neutral and negatively charged terms in the definition of $\varepsilon_{\mathrm{gs}}$ differently. A systematic study of the SIE error effect is beyond the scope of this work. We notice however, that we can obtain an estimate of the SIE through a comparison between B3LYP and periodic Hartree-Fock calculations on the same samples (Table II). From this data, we estimate that, at intermediate and large densities, the SIE in $\varepsilon_{\mathrm{gs}}$ is of the order of $3 \mathrm{eV}$, similar in magnitude to our correction $c$.

Finally, we examine the spatial distribution of the excess electron in the fluid systems to establish whether the delocalisation predicted by the Lanczos method is also observed in DFT calculations. We consider the excess electron ground-state electron density, $\rho^{\mathrm{ex}}(\mathbf{r})=\left|\phi_{\mathrm{gs}}(\mathbf{r})\right|^{2}$, where $\phi_{\mathrm{gs}}$ is the excess electron wave-function at the position $\mathbf{r}$. In Figure 5 we compare $\rho^{\text {ex }}(\mathbf{r})$ obtained from the Lanczos ground-state wave-function $\phi_{\mathrm{gs}}$ and the excess electron density obtained from DFT, $\rho^{\mathrm{ex}}(\mathbf{r})=\rho(N+1 ; \mathbf{r})-\rho(N ; \mathbf{r})$ at the atomic densities $\rho^{*}=0.5$ and 0.9. Both the Lanczos and the DFT excess electron densities are clearly delocalised. The degree of electron localisation has been used $^{38}$ to understand the difference between the variation of electron mobility with density in methane and that in ethane, where the wavefunction becomes localised at liquid densities. A numerical measure of the similarity of the electron densities, $r$, can be obtained from a projection of the DFT excess electron wavefunction (as approximated by the square root of the excess density) onto the Lanczos wavefunction,

$$
r=\left\langle\phi_{g s}^{D F T} \mid \phi_{g s}^{\text {Lancos }}\right\rangle=\iiint d x d y d z \sqrt{\rho_{e x}^{D F T}} \phi_{g s}^{\text {Lanczos }} .
$$

For methane we found $r=0.82 \pm 0.03$ at $\rho^{*}=0.9$ and $r=0.69 \pm 0.02$ at $\rho^{*}=0.5$ from an average over 5 configurations, which indicates that the excess electron distributions in real space are very similarly described by the two methods. We also notice that the presence of the excess electron has the effect of reducing the total electron density in regions close to the methane molecules and increasing it in the free volume between the molecules.

\section{Discussion and Conclusion}

The problem of interpreting the KS orbitals in approximate DFT methods is well known ${ }^{39}$ Error! Bookmark not defined. and it has important implications for the calculation of electron excess energies, especially in condensed-phase systems. Stowasser and Hoffman ${ }^{40}$ used Hartree-Fock (HF) and DFT methods and one-electron extended Hückel calculations to 
study the orbitals and eigenvalues of small molecules, such as $\mathrm{H}_{2} \mathrm{O}$ and $\mathrm{N}_{2}$. They concluded that the KS orbitals are physically reasonable, and that their energies can be made to correspond to occupied and virtual HF orbitals by a scaling of the form $\Delta \varepsilon=a \varepsilon_{\mathrm{HF}}+b$, with $\Delta \varepsilon=\varepsilon_{\mathrm{KS}}-\varepsilon_{\mathrm{HF}}$, where $b$ corrects for the electron self-interaction in DFT and $a$ is the gradient of the linear scaling, with $a$ different for occupied and unoccupied (virtual) orbitals. Salzner and $\mathrm{Baer}^{41}$ proposed a method in which the KS HOMO energy is tuned to be equal to $-I P$, where IP is the first ionisation potential using range-separated hybrids (RSHs) with a rangeparameter tuning procedure within a generalised KS approach which gave good results for small molecules.

For fluid methane we find that no density-independent correction can adjust the DFT orbital energies to approximate the experimentally determined excess-electron lowest-energy conducting state $V_{0}$. This finding is consistent with the fact that, in general, the lowest unoccupied KS orbital energy is not related to the true energy of the conduction band bottom or to the electron affinity of the system.Error! Bookmark not defined. The experimental data for the conduction band energy has two key features: a small variation of a few tenths of an $\mathrm{eV}$ with atomic density and a minimum at an effective density $\rho^{*}=0.7$. DFT calculations fail to reproduce the behaviour of experimental energies, which is on the other hand correctly described by the Lanczos pseudopotential method.

In contrast to the KS orbitals, the ground-state properties of the excess electron calculated with DFT from total energy and electron density differences are qualitatively correct for $\rho^{*}$ higher than 0.3. Furthermore, the agreement can be made quantitative through the addition of a constant (density independent) correction term $c=-2.63 \mathrm{eV}$. We therefore conclude that, at least for delocalised excess electrons, excess electron properties for wide density ranges are more reliably estimated using total energy differences rather than KS orbital energies. The question of whether these results can be extended to excited states, other simple fluid alkanes, like ethane, and more complex disordered materials composed of asymmetric molecules, like amorphous polyethylene, will be the subject of future work.

\section{Acknowledgements}

FS and NQ acknowledge the financial support of EPSRC through grant EP/N002288/1, Advanced Polymer materials for Energy Security - POLYMAT and HPC support through ARCHER project e446. This work was supported by EPSRC through a Service Level Agreement with STFC Scientific Computing Department and by the UK Materials Chemistry Consortium (Grant EP/L000202). 


\section{Figures}

Figure 1 (one column)
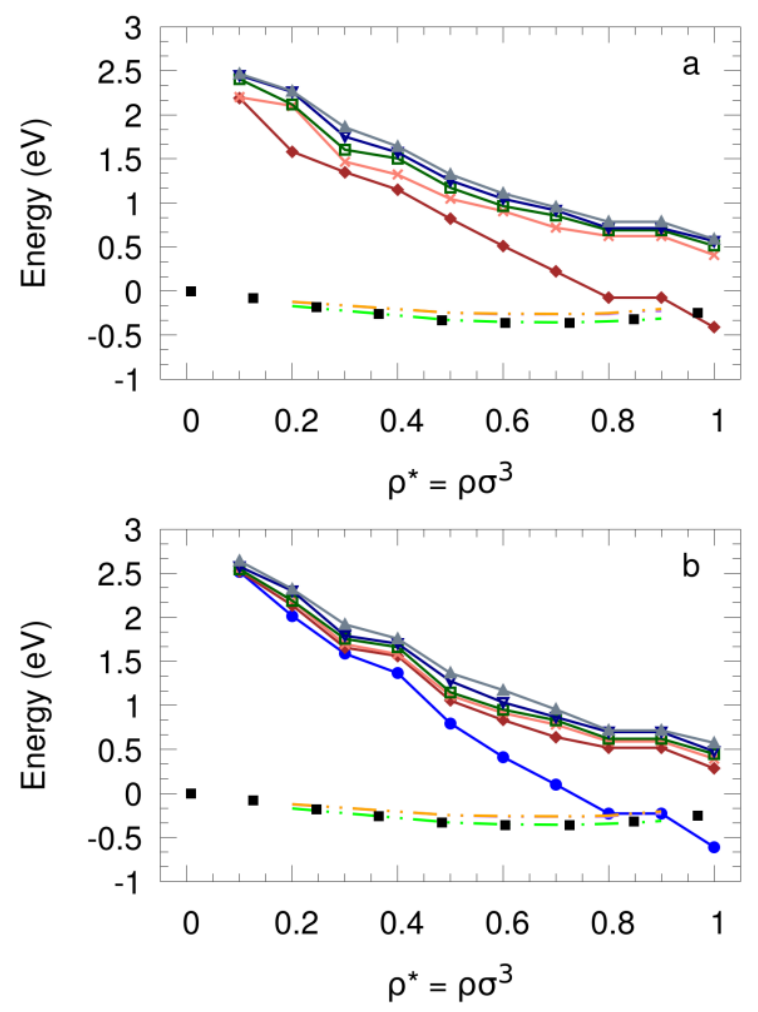

Figure 1. Orbital energies as a function of density for (a) methane ( $N$ electrons) fluid and (b) methane with an excess electron $(N+1$ electrons), compared to the experimental values of the excess-electron lowest-energy conducting state $V_{0}$ at $340 \mathrm{~K}$. The ground state and excited states of the Lanczos excess-electron system are also shown. Experimental energies are shown as filled black squares, Lanczos energies are shown with dashed lines in green for ground state, purple for the first excited state, and green for the second excited state. DFT Kohn-Sham energies are shown with solid lines in filled blue circles for the HOMO, with filled brown diamond for the LUMO, salmon for LUMO+1, dark green unfilled diamonds for LUMO+2, dark blue unfilled inverted triangles for $\mathrm{LUMO}+3$, and grey filled triangles for $\mathrm{LUMO}+4$. The electron concentration was $* * * *$ 
Figure 2 (one column)

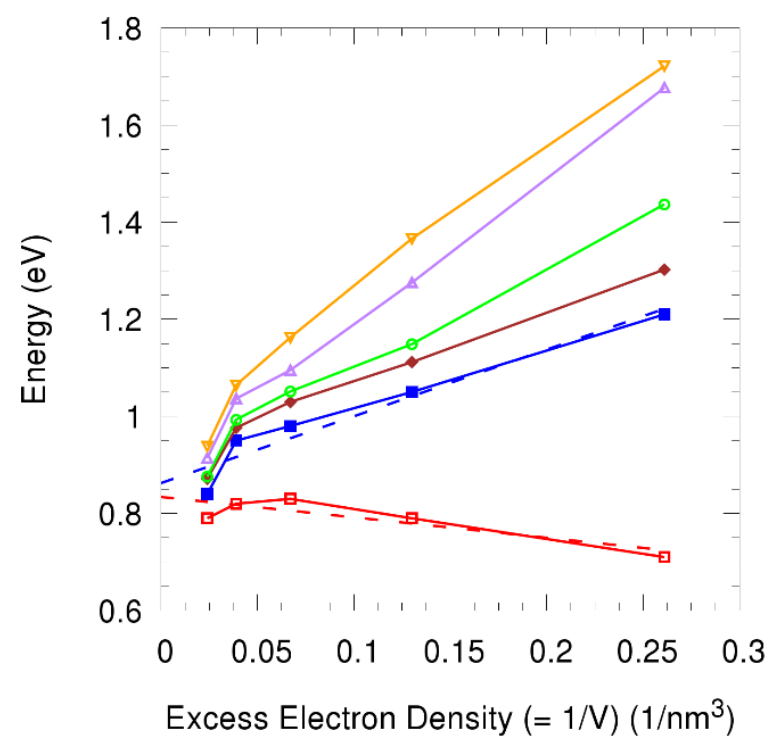

Figure 2. Variation of Kohn-Sham orbital energies with excess-electron density at the atomic density rho* $=0.5$ for the charged system. The lowest curve (unfilled red squares) represents the HOMO energy. Higher energy curves correspond to the LUMO-LUMO+4 energies. The dashed lines show linear fits $E=1.3754 / V+0.8625$ for the HOMO and $E=-0.4201 / V+0.8339$ for the LUMO. 
Figure 3 (one column)

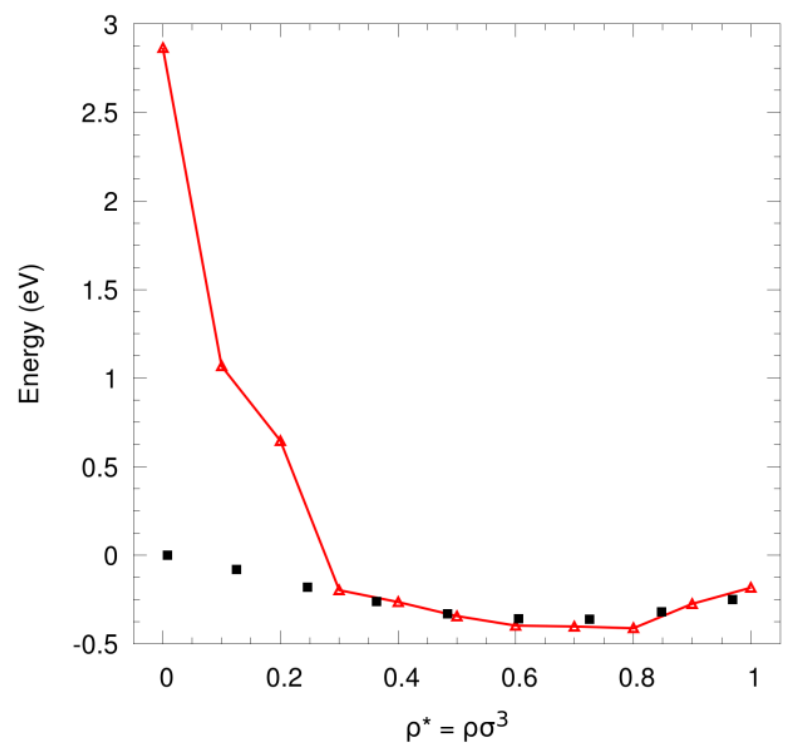

Figure 3. DFT excess electron energy as a function of the effective density calculated from DFT using Equation (1) with the constant correction $\mathrm{c}=-2.63 \mathrm{eV}$ (triangles) and experimental excess electron values (squares). The electron concentration was $* * * *$ 
Figure 4 (one column)

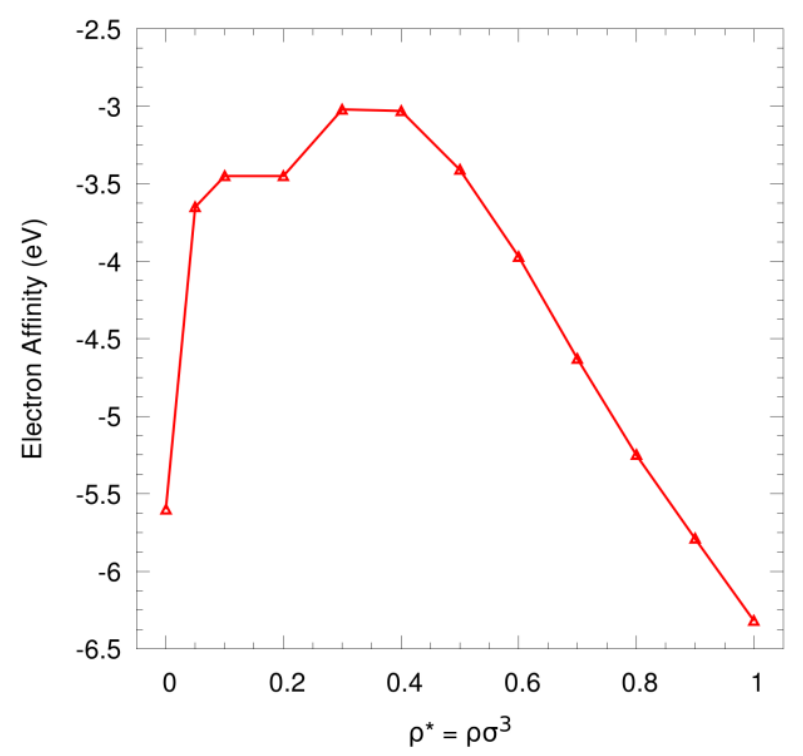

Figure 4. B3LYP/6-31G** electron affinity of a single methane molecule in a periodic cell of length $\mathrm{L} / \sigma$. The value at $\rho^{*}=0$ is for an isolated methane molecule in vacuum (no periodic boundary conditions). 
Figure 5 (one column)

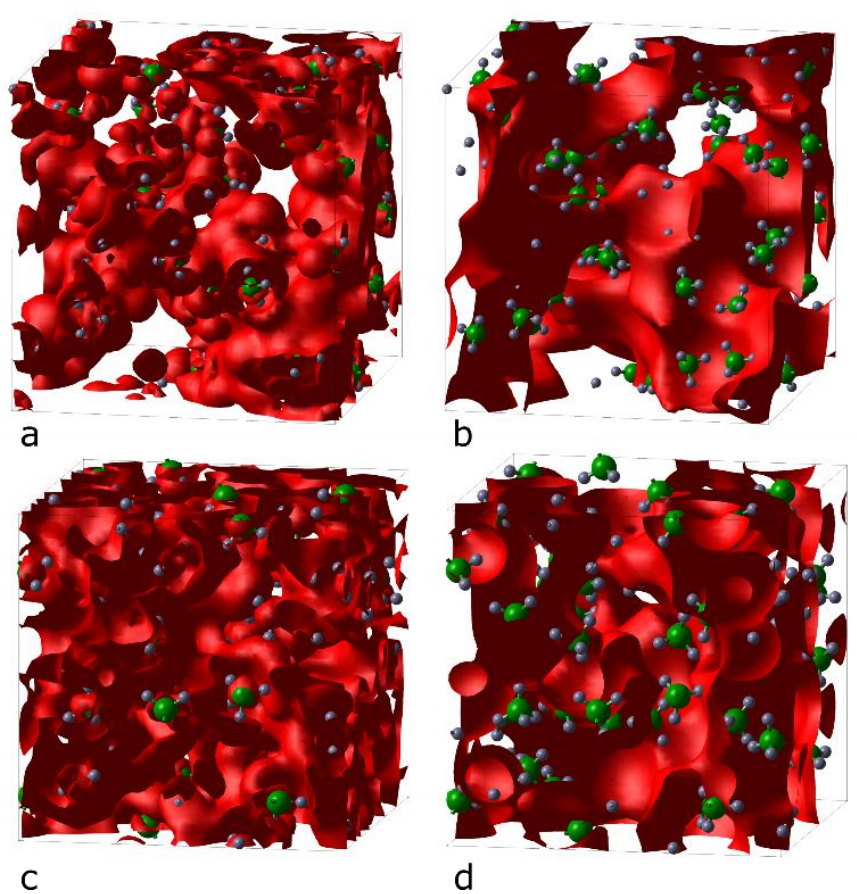

Figure 5. Electron density from the total electron density differences for one configuration at $\rho^{*}=0.5$ with B3LYP/6-31G** (a) and the Lanczos method (b) and $\rho^{*}=0.9$ with B3LYP/6-31G** (c) and Lanczos (d). Red isosurfaces show an electron density of $2.60 \times 10^{-5}$ electron/Bohr ${ }^{3}$ for DFT and 2.55 x $10^{-5}$ for Lanczos with rho* $=0.5$, and $2.91 \times 10^{-5}$ electron/Bohr ${ }^{3}$ for DFT and $3.19 \times 10^{-5}$ for Lanczos with rho* $=0.9$. 


\section{Tables}

Table I

\begin{tabular}{|l|c|}
\hline Basis set & Electron affinity $(\mathrm{eV})$ \\
\hline $6-31 \mathrm{G}^{* *}$ & -5.60 \\
\hline $6-31+\mathrm{G}$ & -1.98 \\
\hline $6-31+\mathrm{G}^{* *}$ & -1.96 \\
\hline $6-31++\mathrm{G}^{* *}$ & -0.95 \\
\hline $6-311++\mathrm{G}^{* *}$ & -0.92 \\
\hline $6-311++\mathrm{G}^{* *}(2 \mathrm{~d}, 2 \mathrm{p})$ & -0.92 \\
\hline
\end{tabular}

Table I. Electron Affinities at $\rho^{*}=0.0$ for a set of selected basis sets collected from the Environmental Molecular Sciences Laboratory (EMSL) website. ${ }^{42,43}$ 
Table II

\begin{tabular}{|l|c|c|c|c|}
\hline & \multicolumn{2}{|c|}{$\rho^{*}=0.5$} & \multicolumn{2}{c|}{$\rho^{*}=0.9$} \\
\hline & neutral & charged & neutral & charged \\
\hline$\Delta \mathrm{HOMO}_{20}(\mathrm{eV})$ & 4.03 & -3.11 & 3.97 & -2.95 \\
\hline$\Delta \mathrm{HOMO}_{30}(\mathrm{eV})$ & 3.41 & -2.86 & 3.36 & -2.75 \\
\hline$\Delta \mathrm{HOMO}_{40}(\mathrm{eV})$ & 2.79 & -2.61 & 2.75 & -2.55 \\
\hline$\Delta \mathrm{HOMO}_{50}(\mathrm{eV})$ & 2.17 & -2.39 & 2.13 & -2.36 \\
\hline & & & & -2.77 \\
\hline$\Delta \mathrm{LUMO}_{20}(\mathrm{eV})$ & -3.33 & -3.18 & -2.48 & -2.93 \\
\hline$\Delta \mathrm{LUMO}_{30}(\mathrm{eV})$ & -3.01 & -2.92 & -2.19 & -2.69 \\
\hline$\Delta \mathrm{LUMO}_{40}(\mathrm{eV})$ & -2.70 & -2.67 & -1.91 & -2.45 \\
\hline$\Delta \mathrm{LUMO}_{50}(\mathrm{eV})$ & -2.39 & -2.43 & & \\
\hline
\end{tabular}

Table II. Energy differences between DFT (B3LYP) and Hartree-Fock treatments of methane at two densities, for the HOMO $\left[\Delta \mathrm{HOMO}=\varepsilon_{\mathrm{DFT}}(\mathrm{HOMO})-\varepsilon_{\text {Hartree-Fock }}(\mathrm{HOMO})\right]$ and LUMO $[\Delta \mathrm{LUMO}=$ $\left.\varepsilon_{\mathrm{DFT}}(\mathrm{LUMO})-\varepsilon_{\text {Hartree-Fock }}(\mathrm{LUMO})\right]$ where $\varepsilon_{\mathrm{DFT}}$ is the HOMO/LUMO orbital energy with a 6$31 \mathrm{G}^{* * 44,45}$ basis set. 


\section{References}

${ }^{1}$ R. A. Holroyd, W. F. Schmidt, Ann. Rev. Phys. Chem. 40, 439 (1989).

${ }^{2}$ Z. Liu and B. J. Berne, J. Chem. Phys. 99, 9054 (1993).

${ }^{3}$ B. Space, D. F. Coker, Z. H. Lui., B. J. Berne, and G. Martyna, J. Chem. Phys. 97, 2002 (1992).

${ }^{4}$ D. Cubero, N. Quirke, and D. F. Coker, J. Chem. Phys. 119, 2669 (2003).

${ }^{5}$ N. A. Deskins, R. Rousseau, and M. Dupuis, J. Phys. Chem. C 114, 5891 (2010).

${ }^{6}$ C. M. Righi, S. Serra, E. Tosatti, S. Scandolo, and G. Santoro, Phys. Rev. Lett. 87, 076802 (2001).

${ }^{7}$ G. Teyssedre, C. Laurent, A. Aslanides, N. Quirke, L. A. Dissado, G. C. Montanari, A. Campus, L. Martinotto, IEEE Trans Dielectrics and Electrical Insulation 8744 (2001).

${ }^{8}$ D. Cubero and N. Quirke, J. Chem. Phys. 120, 7772 (2004).

${ }^{9}$ S. Serra, G. C. Montanari, and L. A. Dissado, J. App. Phys. 116, 224901 (2014).

${ }^{10}$ J. Anta, J Nelson, and N. Quirke, Phys Rev B 60, 125324 (2002).

${ }^{11}$ P. W. Anderson, Phys. Rev. 109 1492, (1958) see also E. Abrahams, P.W Anderson, D.C

Licciardello, and T.V. Ramakrishna, Phys. Rev. Lett. 42, 673 (1979).

${ }^{12}$ L. A. Dissado and J. C. Fothergill, Electrical Degradation and Breakdown in Polymers (P.

Peregrinus, London, 1992).

${ }^{13}$ M. Meunier and N. Quirke, J. Chem. Phys. 113, 369 (2000).

${ }^{14}$ Y. Wang, D. MacKernan, D. Cubero, D. Coker, and N. Quirke, J. Chem. Phys 140, 154902 (2014)

${ }^{15}$ S. Serra, S. Iarlori , E. Tosatti, S. Scandolo, M.C. Righi, and G.E. Santoro, Chem. Phys. Lett. 360, 487 (2002).

${ }^{16}$ R. van Meer, O. V. Gritsenko, and E. J. Baerends, J. Chem. Theory Comput. 10, 4432 (2014).

${ }^{17}$ J. Muscat, A. Wander, and N. M. Harrison, Chem. Phys. Lett. 342, 397 (2001).

${ }^{18}$ L. Bernasconi, S. Tomic, M. Ferrero, M. Rerat, R. Orlando, R. Dovesi, and N. M. Harrison, Phys.

Rev. B 83, 195325 (2011).

${ }^{19}$ S. J. Plimpton, J. Comput. Phys. 117, 1 (1995).

${ }^{20}$ B. Chen and J. I. Siepmann, J. Phys. Chem. B 103, 5370 (1999).

${ }^{21}$ J. Hutter, M. Iannuzzi, F. Schiffmann, and J. VandeVondele, Comput. Mol. Sci. 4, 15 (2014).

${ }^{22}$ J. VandeVondele, M. Krack, F. Mohamed, M. Parrinello, T. Chassaing, and J. Hutter. Comput.

Phys. Commun. 167, 103 (2005).

${ }^{23}$ A. D. Becke, Phys. Rev. A 38, 3098 (1988).

${ }^{24}$ C. Lee, W. Yang, and R. G. Parr, Phys. Rev. B 37, 785 (1988).

${ }^{25}$ S. Goedecker, M. Teter, and J. Hutter, Phys. Rev. B 54, 1703 (1996).

${ }^{26}$ M. Krack, Theor. Chem. Acc. 114, 145 (2005).

${ }^{27}$ J. VandeVondele and J. Hutter, J. Chem. Phys. 127, 114105 (2007).

${ }^{28}$ S. Grimme, J. Comp. Chem. 27, 1787 (2006).

${ }^{29}$ R. Dovesi, R. Orlando, A. Erba, C. M. Zicovich-Wilson, B. Civalleri, S. Casassa, L. Maschio, M.

Ferrabone, M. De La Pierre, P. D’Arco, Y. Noel, M. Causa, M. Rerat, and B. Kirtman, Int. J.

Quantum Chem. 114, 1287 (2014).

${ }^{30}$ T. P. M. Goumans, A. Wander, W. A. Brown, and C. R. A. Catlow, Phys. Chem. Chem. Phys. 9,

2146 (2007).

${ }^{31}$ G. A. Petersson, A. Bennett, T. G. Tensfeldt, M. A. Al-Laham, W. A. Shirley, and J. Mantzaris, J. Chem. Phys. 89, 2193 (1988).

${ }^{32}$ G. A. Petersson and M. A. Al-Laham, J. Chem. Phys. 94, 6081 (1991).

${ }^{33}$ S. Grimme, J. Chem. Phys. 124, 034108 (2006).

${ }^{34}$ A. Ramírez-Solís, Comp. Chem. 2, 31, (2014).

${ }^{35}$ S. Chawla and G. A. Voth, J. Chem. Phys. 108, 4697 (1998)

${ }^{36}$ L. Bernasconi, M. Sprik and J. Hutter, Chem. Phys. Lett. 394, 141 (2004). 
${ }^{37}$ D. Marx and J. Hutter, Ab initio molecular dynamics: Theory and implementation, in: J.

Grotendorst (Ed.), 'Modern Methods and Algorithms of Quantum Chemistry', NIC Series, vol. 1, FZ Jülich, 2000.

${ }^{38}$ Z. Lui and B. Berne, J. Chem. Phys. 99, 9054 (1993).

${ }^{39}$ A. J. Cohen, P. Mori-Sanchez, and W. Yang, Chem. Rev. 112, 289, (2012).

${ }^{40}$ R. Stowasser and R. Hoffmann, J. Am. Chem. Soc. 121, 3414, (1999).

${ }^{41}$ U. Salzner and R. Baer, J. Chem. Phys. 131, 231101 (2009).

${ }^{42}$ D. J. Feller, Comp. Chem. 17, 1571 (1996).

${ }^{43}$ K.L. Schuchardt, B.T. Didier, T. Elsethagen, L. Sun, V. Gurumoorthi, J. Chase, J. Li, and T. L. Windus, J. Chem. Inf. Model. 47, 1045 (2007).

${ }^{44}$ G. A. Petersson, A. Bennett, T. G. Tensfeldt, M. A. Al-Laham, W. A. Shirley, and J. Mantzaris, J. Chem. Phys. 89, 2193 (1988).

${ }^{45}$ G. A. Petersson and M. A. Al-Laham, J. Chem. Phys. 94, 6081 (1991). 